\title{
Influence of number of fusions on mechanical properties of Ducinox alloy
}

\author{
L. Klimek a,b, E. Wołowiec-Korecka a,* \\ a Institute of Material Science and Engineering, Lodz University of Technology, \\ ul. Stefanowskiego 1/15, 90-924 Łódź, Poland \\ b Department of Dental Techniques, Medical University of Lodz, \\ ul. Pomorska 251, 92-231 Łódź, Poland \\ * Corresponding e-mail address: emilia.wolowiec@p.lodz.pl
}

\section{ABSTRACT}

Purpose: The purpose of the test was to specify influence of re-fusions of the nickelchromium prosthetic alloy Ducinox on its strength properties.

Design/methodology/approach: The tests were carried out on the samples cast of the Ducinox alloy molten once, twice and three times. The hardness has been measured using the Vickers method; the tensile strength has been also specified. The samples designed for strength tests were subjected to X-ray control to find possible defects that would eliminate the samples from further tests. The last test was observation of fractures occurred as a result of the strength tests, under the microscope.

Findings: Both hardness measurements and strength tests did not show statistically significant differences among individual groups of samples.

Practical implications: Based on the results of the tests carried out, it can be found that re-fusion of the alloy Ducinox has no influence on its tensile strength. Thereby, in the dental technology practice, it is possible to add the casting scrap to pure metallic alloy without influence on strength of prosthetic element castings.

Originality/value: This is an well-known method of analysis which is applied in many scientific fields. However in modern prosthetics it is new approach.

Keywords: Casting; Fusion; Prosthetic alloys; Mechanical properties

\section{Reference to this paper should be given in the following way:}

L. Klimek, E. Wołowiec-Korecka, Influence of number of fusions on mechanical properties of Ducinox alloy, Journal of Achievements in Materials and Manufacturing Engineering 78/1 (2016) 21-24.

\section{maNUfAGtURING AND PROGESSING}

\section{Introduction}

Mechanical properties of metals used in the dental prosthetics and especially their strength properties are essential to determine their use in prosthetic works. Prosthetic elements are subject to great forces occurring in the oral cavity during chewing. They are accompanied by great stresses so it is important that prosthetic structural 
components have suitable mechanical strength. This condition is met by common metal alloys. Thanks to their mechanical, physical and chemical properties that equal or sometimes exceed these of noble metal alloys as well as low buying costs, the materials are used most often in dental technology.

To reduce material costs at prosthetic laboratories additionally, casting remainders, i.e. cast channels, not cast-in alloy and even metal pieces remaining after prosthetic treatment are used to prepare new castings. The research shows that characteristics of the remolten alloy can be different than these of the "factory" alloy. Both the alloy composition [1] and mechanical properties [2] can undergo changes. Whereat, according to different authors, they can be increased [3] or decreased [4]. Also, the research carried out in several centres have shown influence of alloy remelting on its cytotoxicity [5] and corrosion resistance. Some authors indicate that changes of corrosion resistance after consecutive fusions are omissible $[6,7]$. However, in another studies [8], worsening of corrosive properties of e.g. Wirolloy alloy is found after 3 consecutive fusions. The manufacturers do not recommend use of casting remainders, too; they justify this with changes in chemical composition of repeatedly cast-in alloys. In addition, re-fusion can influence on strength of the connection with porcelain, marginal sealing of crowns, etc. [9]. As you can see, literature reports on remolten alloys are not univocal. There are some studies that deny use of remolten alloys and also some works that demonstrate no influence of re-fusion on the later properties of alloys. Accuracy of metal surface clean-out before re-fusion has great influence on mechanical properties of the remolten alloy [10-12].

The purpose of the research shown in this publication was to specify influence of re-fusions of the nickel-chromium prosthetic alloy Ducinox on its strength properties.

\section{Materials and methods}

Samples are made of nickel-chromium alloy Ducinox from Ugin' dentaire. The output alloy composition specified with the fluorescent X-ray spectrometry method using the spectrometer SRS 303 is shown in Table 1. The alloy has been molten in a crucible with a gas burner. The samples have been cast-in using the centrifugal method in the casting centrifuge WR-01 from Prod-Dent and cooled with the mould in the room temperature $\left(21^{\circ} \mathrm{C}\right)$. The tests were carried out on the samples made of the alloy molten once, twice and three times. All samples have been made at the same laboratory by the same technician to ensure the same conditions for alloy preparation. The cylindrical and rod- shaped samples were tested and their chemical composition is shown in the Table 1.

Table 1.

Chemical composition of the tested alloy

Percentage content of elements ( $\%$ by weight)

\begin{tabular}{cccccc}
\hline $\mathrm{Fe}$ & $\mathrm{Ni}$ & $\mathrm{Cr}$ & $\mathrm{Mo}$ & $\mathrm{Mn}$ & $\mathrm{Si}$ \\
\hline balanced & 25.79 & 22.87 & 3.11 & 1.47 & 2.75 \\
\hline
\end{tabular}

The cylindrical samples of diameter $12 \mathrm{~mm}$ and height $7 \mathrm{~mm}$ were subject to hardness tests. The hardness has been measured using the Vickers method at the load $100 \mathrm{~N}$ (10 KG) on five samples from each fusion. For strength tests, the rod-shaped samples of diameter $3 \mathrm{~mm}$ and length $40 \mathrm{~mm}$ were used after turning in the central part to the diameter $2 \mathrm{~mm}$ along the length $15 \mathrm{~mm}$ (Fig. 1). X-ray analysis of each rod was performed in order to control the cast quality. The test has been carried out by means of head X-ray apparatus BALTOSOT GFD165 HAND-X. The samples in which porosity and blowholes were detected, have been removed from the further tests.

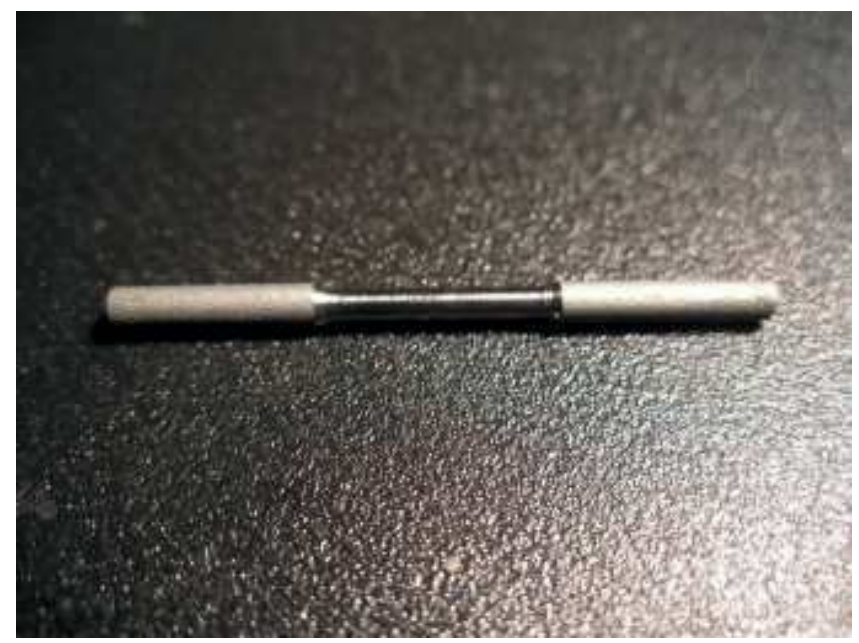

Fig. 1. A sample for strength tests

The casting defects have been evaluated based on the standard PN-EN 12681 (Founding. Radiographic examination). Strength tests have been carried out on the testing machine Zwick-Rooel Z100. Five samples from each fusion have been tested. To characterize types of broken sample fractures and to detect possible casting defects in the fracture area, they were subject to fractographic tests by means of the scanning electron microscope HITACHI S-3000N. 


\section{Results}

The results of the hardness measurements are shown in Table 2. Results of the strength tests are given in Table 3 while their graphic image is shown in Fig. 2.

Table 2.

Hardness HV10 of the tested samples

\begin{tabular}{cccc}
\hline & \multicolumn{3}{c}{ Number of fusions } \\
\cline { 2 - 4 } & one & two & three \\
\hline HV10 & 141 & 142 & 138 \\
\hline Standard deviation & 7 & 8 & 8 \\
\hline
\end{tabular}

Table 3.

Results of the strength tests

\begin{tabular}{cccc}
\hline & \multicolumn{3}{c}{ Number of fusions } \\
\cline { 2 - 4 } & one & two & three \\
\hline $\mathrm{Rm}-$ mean, $\mathrm{MPa}$ & 474 & 492 & 472 \\
\hline Standard deviation & 36 & 30 & 10 \\
\hline
\end{tabular}

a)

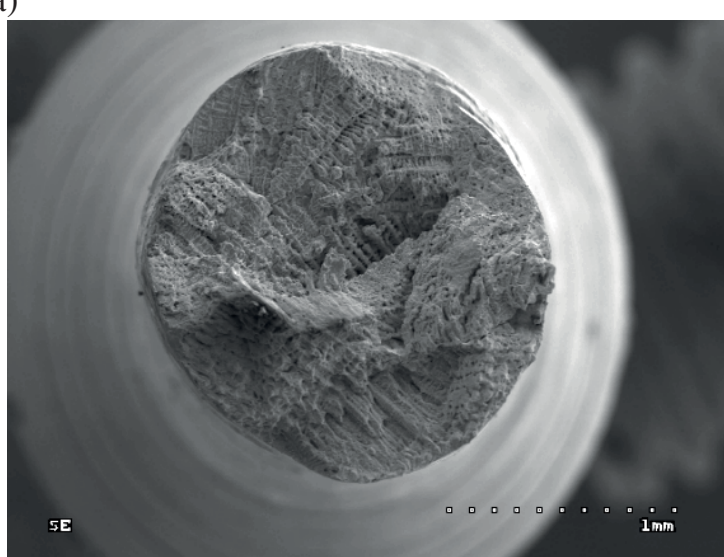

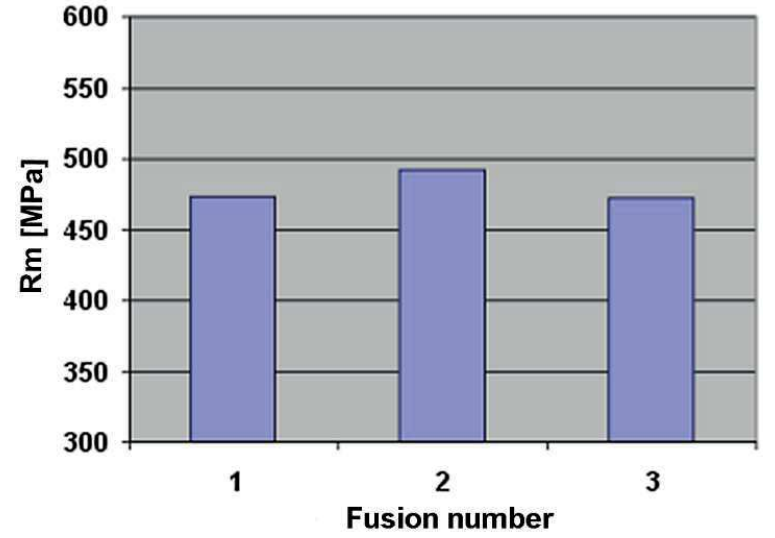

Fig. 2. Graphic image of strength results for alloys after the tensile test

The exemplary sample fractures received after the tensile test are shown in Figs. 3-5.

b)

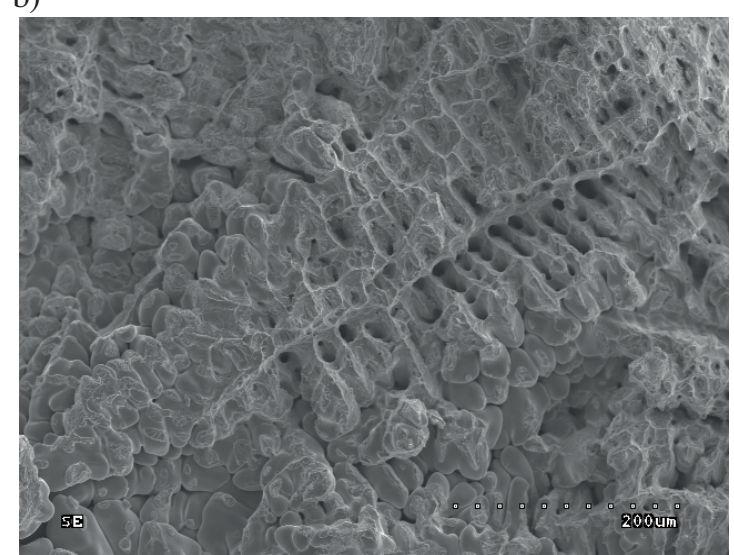

Fig. 3. Sample fractures after the single fusion. a) the macro view, b) the enlarged fracture fragment

a)

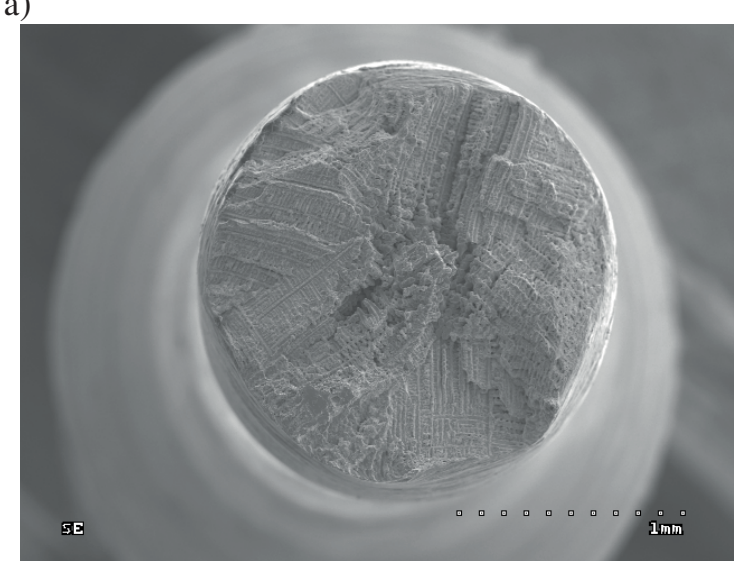

b)

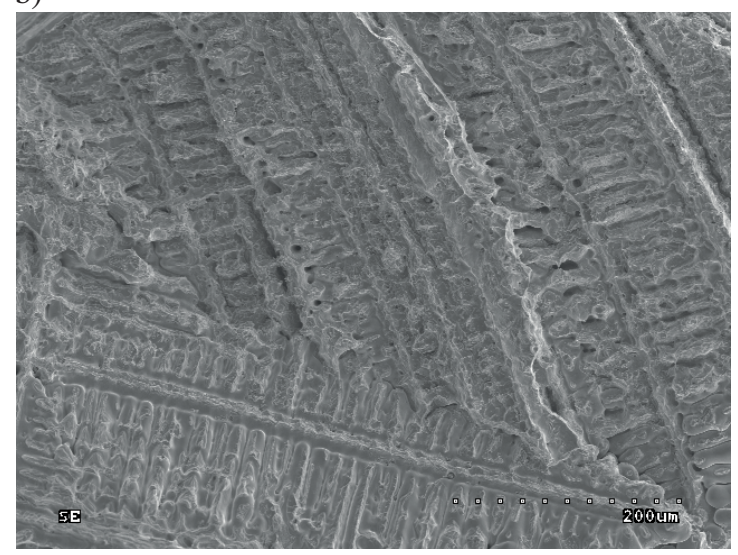

Fig. 4. Sample fractures after the double fusion. a) the macro view, b) the enlarged fracture fragment 
a)

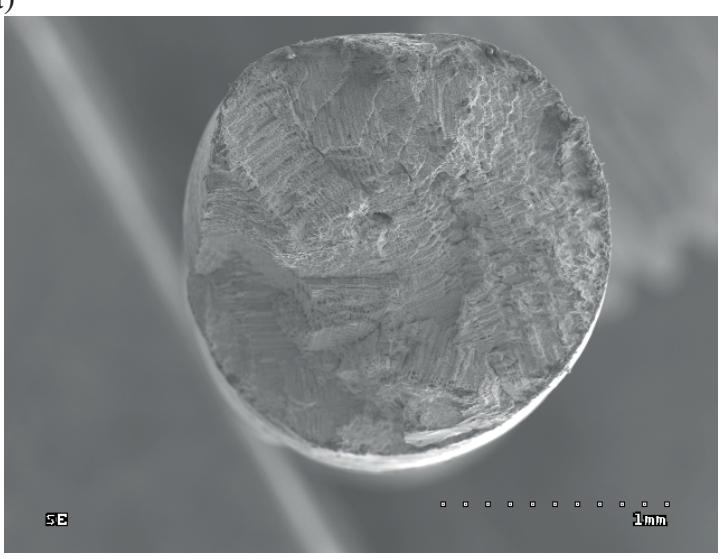

b)

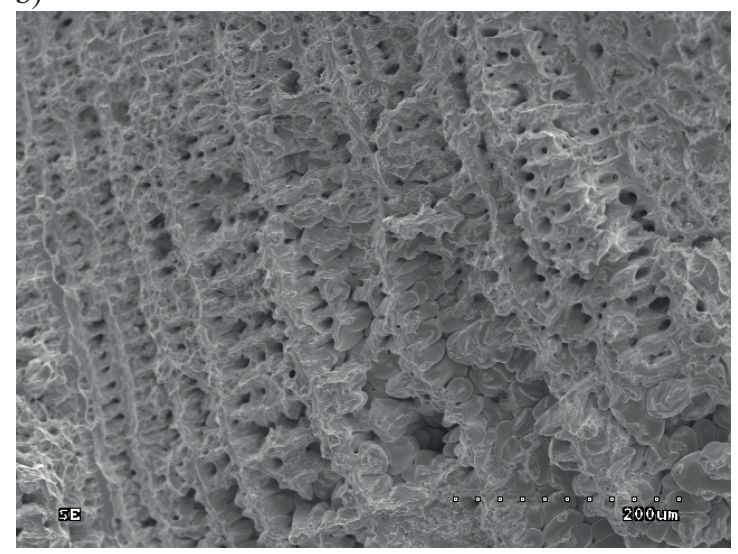

Fig. 5. Sample fractures after the triple fusion. a) the macro view, b) the enlarged fracture fragment

\section{Discussion}

The results of the hardness measurements for the alloys received after different number of fusions are slightly different, however the differences are not statistically significant. Also, tensile strength of the samples from individual groups is different, however the statistical analysis has not shown statistically significant differences. So, it can be expected that the alloy re-fusion does not affect its mechanical properties.

The sample fractures received after the tensile test (Figs. 2-5) are similar in each of the groups involved. In all cases, these are the interdendritic fractures (cracking occurs between the original alloy dendrites). The sample cracking character is mostly brittle.

\section{Conclusions}

Based on the results of the tests carried out, it can be found that re-fusion of the alloy Ducinox has no influence on its tensile strength.

\section{References}

[1] M. Peraire, J. Martinez-Gomis, J.M. Anglada, J. Bizar, J. Salsench, F.J. Gil, Effects of recasting on the chemical composition, microstructure, microhardness, and ion release of 3 dental casting alloys and titanium, The International Journal of Prosthodontics 20 (2007) 286-288.

[2] S. Majewski, W. Opoka, S. Gacek, Properties of the training alloy depending on the initial ingredients form and the casting multiple, Prosthetics XLI 4 (1991) 192-198.

[3] N. Horasawa, M. Marek, The effect of recasting on corrosion of a silver.-palladium alloy, Dental Materials 20 (2004) 352-357.

[4] G. Henriques, S. Consani, J.M.D. de Almeida Rollo, F.A. e Silva, Soldering and remelting influence on fatigue strength of cobalt-chromium alloys, The Journal of Prosthetic Dentistry 78 (1997) 146-152.

[5] A.S. Al-Hiyasat, Homa Darmani, The Journal of Prosthetic Dentistry 93/2 (2005) 158-163.

[6] E. Khamis, M. Seddik, Corrosion evaluation of recasting non-precious dental alloys, International Dental Journal 45 (1995) 209-217.

[7] S. Ozdemir, A. Arikan, Effects of recasting on the amount of corrosion products released from two Ni-Cr base metal alloys, European Journal of Prosthodontics and Restorative Dentistry 6 (1998) 149-153.

[8] M.A. Ameer, E. Khamis, M. Al-Motlaq, Electrochemical behaviour of recasting $\mathrm{Ni}-\mathrm{Cr}$ and $\mathrm{Co}-$ Cr non-precious dental alloys, Corrosion Science 46 (2004) 2825-2836.

[9] Y. Ucar, Z. Aksahin, C. Kurtoglu, Metal ceramic bond after multiple castings of base metal alloy, The Journal of Prosthetic Dentistry 102 (2009) 165-171.

[10] M.B. Lopes, S. Consani, M.A.C. Sinhoreti, L. CorrerSobrinho, Influence of recasting palladium-silver alloy on the fit of crowns with different marginal configurations, The Journal of Prosthetic Dentistry 94/5 (2005) 430-434.

[11] D.R. Nelson, J.F. Palik, H.F. Morris, M.C. Comella, Recasting a nickel-chromium alloy, The Journal of Prosthetic Dentistry 55 (1986) 122-127.

[12] PN-EN 12681 Founding. Radiographic examination. 